\title{
Quadrant Dynamic with Automatic Plateau Limit Histogram Equalization for Image Enhancement
}

\author{
P. Jagatheeswari, ${ }^{1}$ S. Suresh Kumar, ${ }^{2}$ and M. Mary Linda ${ }^{1}$ \\ ${ }^{1}$ Ponjesly College of Engineering, Nagercoil, Tamil Nadu 629003, India \\ ${ }^{2}$ Sun College of Engineering and Technology, Nagercoil, Tamil Nadu 629902, India \\ Correspondence should be addressed to P. Jagatheeswari; jagatheeswarissk@gmail.com
}

Received 4 April 2014; Revised 5 September 2014; Accepted 7 September 2014; Published 15 October 2014

Academic Editor: Hongyong Zhao

Copyright (c) 2014 P. Jagatheeswari et al. This is an open access article distributed under the Creative Commons Attribution License, which permits unrestricted use, distribution, and reproduction in any medium, provided the original work is properly cited.

\begin{abstract}
The fundamental and important preprocessing stage in image processing is the image contrast enhancement technique. Histogram equalization is an effective contrast enhancement technique. In this paper, a histogram equalization based technique called quadrant dynamic with automatic plateau limit histogram equalization (QDAPLHE) is introduced. In this method, a hybrid of dynamic and clipped histogram equalization methods are used to increase the brightness preservation and to reduce the overenhancement. Initially, the proposed QDAPLHE algorithm passes the input image through a median filter to remove the noises present in the image. Then the histogram of the filtered image is divided into four subhistograms while maintaining second separated point as the mean brightness. Then the clipping process is implemented by calculating automatically the plateau limit as the clipped level. The clipped portion of the histogram is modified to reduce the loss of image intensity value. Finally the clipped portion is redistributed uniformly to the entire dynamic range and the conventional histogram equalization is executed in each subhistogram independently. Based on the qualitative and the quantitative analysis, the QDAPLHE method outperforms some existing methods in literature.
\end{abstract}

\section{Introduction}

An important challenge in the field of digital image processing is contrast enhancement. Compared to the original image, contrast enhancement produces better image by changing the pixel intensities. Of the many techniques available for image contrast enhancement, histogram equalization (HE) is a widely used technique. The fundamental idea of histogram equalization is to flatten the histogram and stretch the dynamic range of the gray levels by using the cumulative density function of the image. Nowadays, histogram equalization is applied in various applications such as medical image processing and radar image processing [1]. In histogram equalization, the brightness of an input image is significantly changed and causes undesirable artifacts. This is not suitable for some applications where brightness preservation is necessary. To overcome the aforementioned problem, several brightness preserving methods have been proposed [2-5].
Generally, these enhancement methods can be classified into two types: partitioned histogram equalization (PHE) and dynamic partitioned histogram equalization (DPHE). In these methods, the original histogram is divided into several subhistograms based on the histogram statistical information. The difference between the PHE and DPHE is that, in the DPHE, each subhistogram is assigned to a new enhanced dynamic range instead of using the original dynamic range. One of the popular PHE based methods is mean brightness preserving bihistogram equalization (BBHE). BBHE segments the original histogram into two portions by the mean of the input histogram. BBHE has been analyzed both experimentally and mathematically that this technique is capable of achieving the brightness preservation [1]. Later, dualistic subimage histogram equalization (DSIHE) has been proposed. This algorithm separates the input image's histogram into two subhistograms based on median of the input image [2]. This technique has been claimed to outperform BBHE 
in terms of both brightness preservation and also entropy (image content) preservation. BBHE and DSIHE methods can preserve the mean brightness of original image to some extent. In order to provide scalable brightness preservation, recursive mean-separate histogram equalization (RMSHE) and minimum mean brightness error bihistogram equalization (MMBEBHE) have been proposed [3].

In RMSHE algorithm, the original image is recursively segmented into some subimages based on their mean brightness and equalized them separately. The better mean brightness will be preserved when the recursive degrees have been increased. If the recursive levels tend to infinity, the output image will be equal to input image [3].

For DPHE, there are two methods existing in literature: dynamic histogram equalization (DHE) [6] and brightness preserving dynamic histogram equalization (BPDHE) [7]. The DHE partitions the histogram of input image based on local minimal gray level and assigns a new dynamic range for each subhistogram. To ensure many dominating portions, the DHE further segments the large subhistogram through a repartitioning test. As the DHE does not consider the mean brightness preservation, it neglects the mean brightness preserving and tends to intensity saturation artifact.

To overcome the drawbacks of the DHE, brightness preserving dynamic histogram equalization (BPDHE) has been introduced [7]. The BPDHE uses the local maximal gray level as the separating point rather than the local minimal gray level. For this reason, Ibrahim and Kong [7] claim that choosing the local maximal gray levels are better for mean brightness preservation. Finally in BPDHE, histogram equalization is implemented after assigning a new dynamic range for each subhistogram. In order to maintain the mean brightness, brightness normalization is applied to ensure that the enhanced image has similar mean brightness to that of the input image.

Research by Praveen and Sathik [8] has done comparison between original image and image enhanced by histogram equalization and contrast limited adaptive histogram equalization (CLAHE) for bone fracture crack detection using pixel value measurement. They concluded that CLAHE is better than histogram equalization. CLAHE had been claimed also to improve the contrast better in the application of automated segmentation of blood vessels in retinal images when compared to histogram equalization [9].

Kokufuta and Maruyama [10] described an approach for real time processing of the contrast limited adaptive histogram equalization (CLAHE) using an FPGA. In this approach, a histogram is generated for each pixel in an image for remapping the pixel, and each histogram is speculatively distributed without iterations by feeding back the distribution result of its previous pixel. But the computational complexity of this approach is very high $[10,11]$.

Ooi et al. [12] proposed a bihistogram equalization plateau limit (BHEPL) as the hybrid of the BBHE and clipped histogram equalization. BHEPL divides the histogram of input image by the value of mean brightness of the input image. Both subhistograms are clipped by each mean of histograms of the occupied intensity, respectively. At last, conventional histogram equalization methods are able to control the enhancement rate. Also these methods can avoid overamplification of noise in the image.

Recently, Wayalun [13] presented an enhancement algorithm for chromosome images based on histogram equalization ( $\mathrm{HE})$. Underwater imaging is quite challenging in the area of photography especially for low resolution and ordinary digital camera. Mean values of the stretched histogram are used to improve the contrast of the image [14]. An advance multiband satellite colour, contrast improvement technique of a poor-contrast satellite images, is proposed based on discrete cosine transform (DCT) in [15], the retinal images are preprocessed using adaptive histogram equalization (AHE), and the blood vessels are enhanced by applying top-hat and bottom-hat transforms in [16]. Based on the inspiration of the local contrast range transform, a new general form of fast dynamic range compression with a localcontrast-preservation (FDRCLCP) algorithm is developed to resolve image enhancement problems directly in the spatial domain [17]. Although these methods perform well for image enhancement, the enhancement process requires high computational costs with a large memory requirement, usually leading to an inefficient algorithm and requiring hardware acceleration.

In this paper, a modified method is proposed as the extension of the BHEPL and RMSHE, called quadrant dynamic with automatic plateau limit histogram equalization (QDAPLHE). First, the input image is passed through a median filter to remove the noises present in the image. Then the proposed method divides the histogram of the filtered image into four subhistograms while maintaining second separated point as the mean brightness. Then the clipping process is implemented by automatically calculating the plateau limit as the clipped level. The clipped portion of the histogram is modified to reduce the loss of image intensity value. Finally, the clipped portion is redistributed uniformly to the entire dynamic range and the conventional histogram equalization is executed in each subhistogram independently. The rest of this paper is organized as follows. In Section 2, the methodology of the proposed quadrant dynamic with automatic plateau limit histogram equalization (QDAPLHE) will be discussed in detail. Then, Section 3 presents the qualitative and quantitative analysis of existing and proposed methods. Finally, Section 4 serves as the conclusion of this work.

\section{Quadrant Dynamic with Automatic Plateau Limit Histogram Equalization (QDAPLHE)}

The proposed QDAPLHE method uses the fundamental idea of the RMSHE. In RMSHE the number of decomposed subhistograms increases in powers of two. Although it will produce better brightness preservation, it declines the effectiveness of the histogram equalization and yields an output image without a good enhancement; that is, the output will be the same as the input. Thus in this work, the input histogram is divided into four subhistograms. The proposed QDAPLHE consists of four processes, namely, filtering process, histogram partitioning process, clipping process, redistribution process, and histogram equalization. 
2.1. Filtering Process. In many signal and image processing applications, it is necessary to smooth the noisy signals while at the same time preserving the edge information. The most commonly used smoothing techniques are linear filtering, averaging filtering, and median filtering. The linear filters smooth the noisy signals and also the sharp edges.

2.1.1. Median Filtering. A median filter finds the median of a number of elements at its input. In the standard median filtering applications, the size of the window is $W$, where $W$ is odd. It is moved along the sampled values of the image or the signal. For each position of the window, the median of the elements within the windows are computed and then written at the output pixel located at the same position as the central element of the window. The median computed at this operation is called the running or the moving median. Since the size of the window is constant, the number of incoming elements is equal to the number of outgoing elements [18]. The dimensions of the filter mask must be odd. Mask sizes may be $3 \times 3,5 \times 5$, or $7 \times 7$. In many cases minimum mask size is preferred. In this paper, the mask size chosen for analysis is $3 \times 3$.

2.1.2. Padding. When the center of the mask moves closer to the border, one or more rows or columns of the mask will be located outside the image plane. There are several ways to handle this situation. One such approach is padding. Padding is the process of adding rows and columns of " 0 "s. Padding is stripped off at the end of the process so that the size of the filtered image is the same as the original image.

2.2. Histogram Partitioning Process. Each image has different histogram, which depends on the brightness and darkness of the image (intensity value), and this histogram is partitioned to enhance the image. The proposed QDAPLHE method divides the histogram into four subhistograms based on mean value. The mean-based partition approach tends to segment the number of pixels equally in each subhistogram. Hence, each separating point can be calculated using the following equations:

$$
\begin{gathered}
s_{1}=0.25 \times\left\{I_{w} \times I_{h}\right\}, \\
s_{2}=0.5 \times\left\{I_{w} \times I_{h}\right\}, \\
s_{3}=0.75 \times\left\{I_{w} \times I_{h}\right\},
\end{gathered}
$$

where $s_{1}, s_{2}$, and $s_{3}$ are intensities set to $0.25,0.50$, and 0.75 , respectively, for the total number of pixels in the histogram of the input image. $I_{w}$ and $I_{h}$ represent the width and height of the input image, respectively.

2.3. Clipping Process. Histogram equalization stretches the high contrast region of the histogram and compresses the low contrast region of the histogram [19]. As a consequence, when the object of interest in an image occupies only a small portion of the image, then the object will not be successfully enhanced by histogram equalization. Histogram equalization method causes level saturation effects as it extremely pushes the intensities towards the right or the left side of the histogram.

A clipped histogram equalization method tends to overcome these problems by restricting the enhancement rate. It is known that the enhancement from histogram equalization is heavily dependent on the cumulative density function $[c(x)]$. Therefore, the enhancement rate is proportional to the rate of $c(x)$. The rate of $c(x)$ is given by

$$
\frac{d}{d x} c(x)=p(x) .
$$

The probability density function $p(x)$ is given by

$$
p(x)=\frac{h(x)}{N},
$$

where $h(x)$ is the histogram for intensity value " $x$ " and " $N$ " is the total number of pixels in the image. The enhancement rate is limited by limiting the value of $p(x)$ or $h(x)$ [20].

Therefore, the clipped histogram equalization modifies the shape of input histogram by decreasing or increasing the value in the histogram's bins based on a threshold limit before the equalization takes place. This threshold limit is also known as the clipping limit or clipping threshold $\left(T_{c}\right)$ or the plateau level of the histogram and based on this threshold value, the histograms will be clipped.

To avoid the intensity saturation and overenhancement problem, the proposed QDAPLHE method adopts the clipped histogram equalization (CHE) to control the enhancement rate by defining plateau limits automatically to each subhistogram. Here, the plateau limit (or $T_{c}$ ) is determined automatically by calculating the average occupied intensity in each subhistogram.

Each plateau limit is identified as follows:

$$
P_{i}=\frac{1}{s_{i}-s_{i-1}} \times \sum_{k=m_{i-1}}^{m_{i}} h\left(X_{k}\right),
$$

where $h\left(X_{k}\right)$ is the histogram at the intensity level " $k$ ".

Clipping process is applied after finding this plateau limit. The clipped portion can be determined as follows:

$$
h_{\mathrm{ci}}\left(X_{k}\right)=\left\{\begin{array}{cc}
h\left(X_{k}\right) & h\left(X_{k}\right) \leq P_{i} \\
P_{i} & h\left(X_{k}\right)>P_{i}
\end{array}\right\},
$$

where $h_{\mathrm{ci}}\left(X_{k}\right)$ is the clipped histogram at intensity level " $k$ ".

While using the clipping process, all the values above the plateau limit $\left(T_{c}\right)$ are removed, which may lead to loss on original intensity value of the image. Hence, the histogram in the clipped portion is adjusted using (6) as

$$
h_{\mathrm{mci}}\left(X_{k}\right)=\left\{\begin{array}{cc}
h_{\mathrm{ci}}\left(X_{k}\right) & h_{\mathrm{ci}}\left(X_{k}\right) \leq P_{i} \\
P_{i}+\frac{h_{\mathrm{ci}}\left(X_{k}\right)-P_{i}}{3} & h_{\mathrm{ci}}\left(X_{k}\right)>P_{i}
\end{array}\right\},
$$

where $h_{\mathrm{mci}}\left(X_{k}\right)$ is the modified clipped histogram at intensity level " $k$ ". This type of clipping process has the effect of the contrast enhancement and reduces the noise amplifying artifacts. 


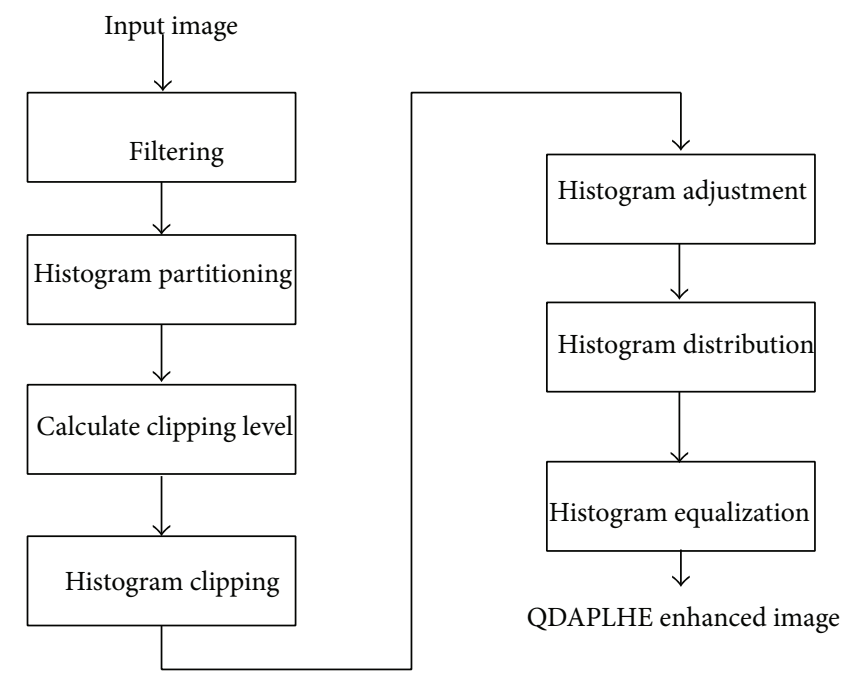

FIGURE 1: Block diagram of proposed QDAPLHE method.

2.4. Redistribution Process. Because of this clipping process, the subhistograms may not ensure the balance space in each subhistogram for sufficient contrast enhancement. When the side of the subhistogram is narrow, contrast enhancement obtained in narrow stretching space is less significant and wide stretching space introduces redundant contrast enhancement. Consequently, the processed image tends to suffer from loss of image details and intensity saturation artifact. To overcome these drawbacks, the clipped portion is redistributed over the entire dynamic range. For this, the proposed QDAPLHE method maintains the point $s_{2}$ as the brightness preserving. The separating points of $s_{1}, s_{2}$, and $s_{3}$ are reassigned to a new grey level represented as $t_{1}, t_{2}$, and $t_{3}$, respectively. Consider

$$
\begin{gathered}
t_{1} \cong s_{2} \times \frac{s_{1}-s_{0}}{s_{2}-s_{0}}, \\
t_{2} \cong s_{2}, \\
t_{3} \cong\left(L-1-s_{2}\right) \times \frac{s_{3}-s_{2}}{s_{4}-s_{2}}+s_{2},
\end{gathered}
$$

where $s_{0}$ and $s_{4}$ are assigned to the minimum and maximum output intensity value (i.e., $s_{0}=0$ and $s_{4}=L-1$ (255)). The new dynamic ranges are determined for all the quadrant subhistograms.

2.5. Histogram Equalization. The final step in the proposed QDAPLHE method is to equalize each new quadrant subhistogram independently. The output of histogram equalization $Y(X)$ of this subhistogram can be determined by using (8). Consider

$$
Y(X)=t_{i-1}+\left(t_{i}-t_{i-1}\right) \times \frac{\sum_{k=m_{i-1}}^{m_{i}} h_{\mathrm{mci}}\left(X_{k}\right)}{m_{i}}
$$

The total of the clipped histogram at ith subhistogram $M_{i}$ is given by

$$
M_{i}=\sum_{k=m_{i-1}}^{m_{i}} h_{\mathrm{mci}}\left(X_{k}\right)
$$

2.6. Step-by-Step Procedure for Developing the Proposed QDAPLHE Method. By combining the above said five processes, the algorithm is developed and the step-by-step procedure to implement the proposed QDAPLHE technique is given in Figure 1.

At first, the input image is taken and the impulse noises present in this image are filtered using median filter. The filtered image is then passed to the histogram partitioning process where the image histogram is partitioned in to four subhistograms using (1). In each subhistogram, to avoid the over enhancement problem, clipping of histogram process is performed. For this, initially the clipping levels (plateau limits) for each subhistogram are calculated using (4). Depending on the clipping level in each subhistogram, histogram clipping is performed using (5).

Due to this clipping process, some information in histogram will be lost and to avoid this problem, histogram adjustment process is performed using (6). The clipped portion is redistributed over the entire dynamic range using (7). Finally, histogram equalization is performed using (8).

\section{Experimental Results and Discussions}

The performance of the proposed QDAPLHE method is tested in numerous images. The images like Einstein, girl, house, couple, copter, F16, and jet are taken from database (http:// decsai.ugr.es/cvg/dbimagenes/) and CT chest, CT brain, and CT abdomen images were obtained from Kanyakumari Government Medical College, Asaripallam, Tamil Nadu, India, with the help of Dr. J.Ravindran. In this work three out of 


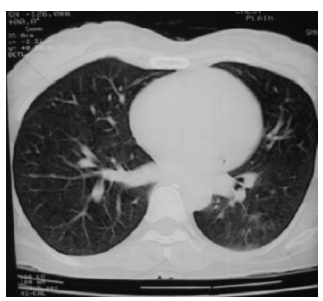

(a) Original

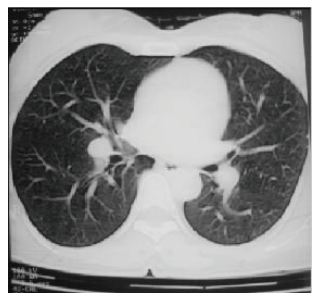

(b) HE

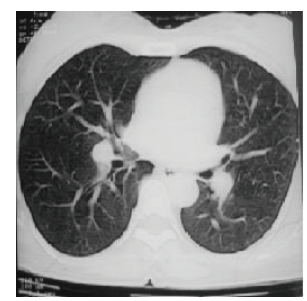

(c) BBHE

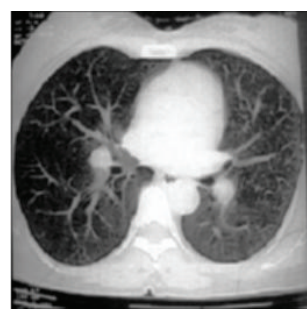

(d) DSIHE

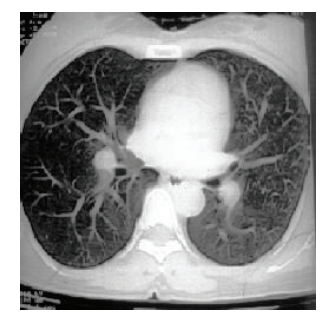

(e) MMBEBHE

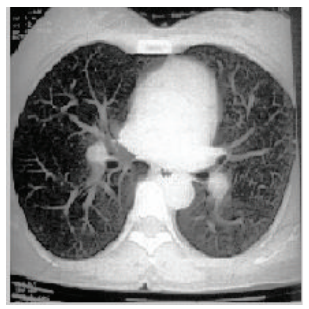

(f) RMSHE

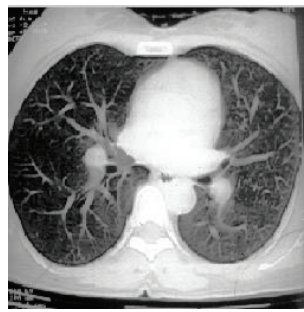

(g) BPDHE

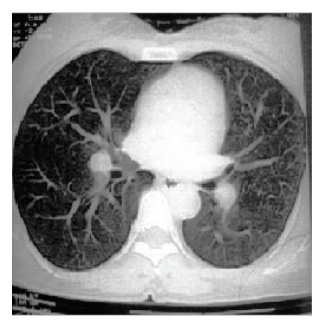

(h) BHEPL

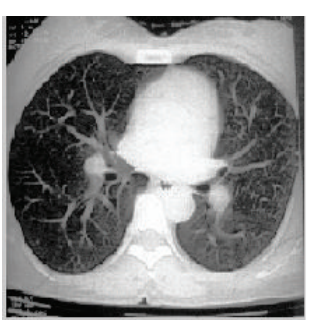

(i) Proposed QDAPLHE

FIgUre 2: Results for CT chest image.

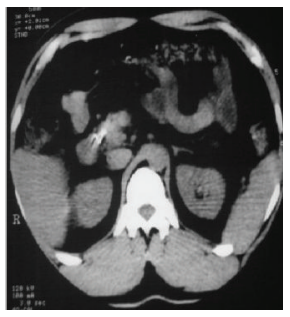

(a) Original

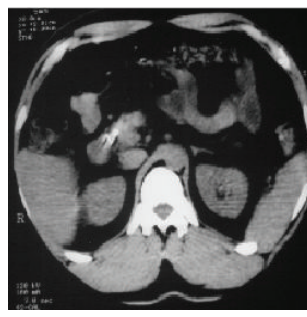

(b) HE

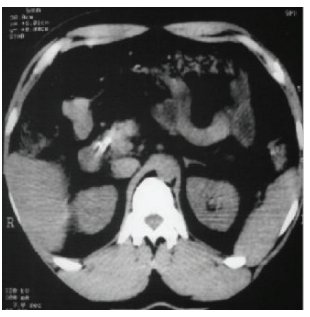

(c) BBHE

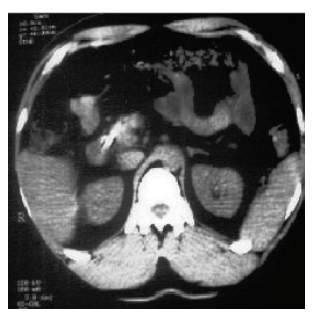

(d) DSIHE

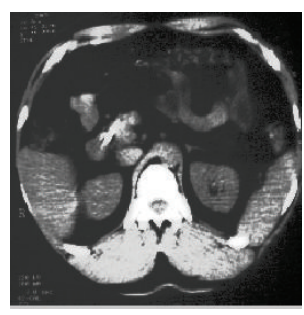

(e) MMBEBHE

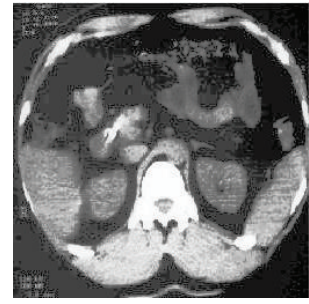

(f) RMSHE

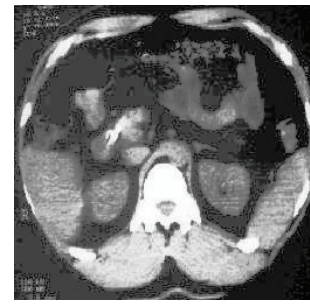

(g) BPDHE

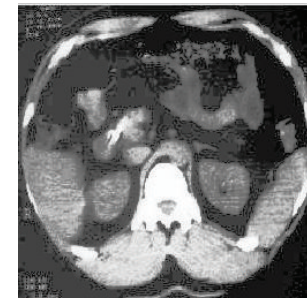

(h) BHEPL

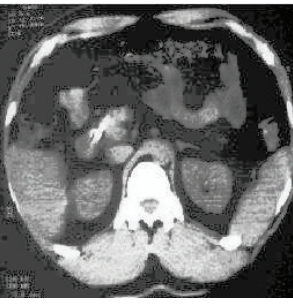

(i) Proposed DAPLHE

FIgURE 3: Results for CT abdomen image.

these sampling images of size $256 \times 256$ pixels, namely, CT chest, CT brain, and CT abdomen are selected to evaluate the capability of the proposed method. The proposed QDAPLHE method is qualitatively and quantitatively analyzed.

3.1. Qualitative Analysis. The qualitative analysis involves performance comparison with existing brightness preserving methods, namely, HE, BBHE, DSIHE, MMBEBHE, RMSHE, BPDHE, and BHEPL. Figures 2, 3, and 4 show the output images produced by these HE methods for the CT chest, CT abdomen, and CT brain image.

From the experimental results, the enhancement produced by existing methods and proposed method are shown in Figures 2(a)-2(i), 3(a)-3(i), and 4(a)-4(i) for various input images. In general, the conventional histogram equalization algorithms are prone to missing luminance levels due to the mapping function calculation and this will lead to cause the lack of information in case of a gradation background. To overcome these shortcomings, clipping based method (QDAPLHE) is developed and the output results are shown in Figures 2(i), 3(i), and 4(i).

Based on Figures 2(b), 3(b), and 4(b), it is clear that the histogram equalization method enhances the images, but it also amplifies the noise level of the images. From Figures 2, 3, 4(c), and 4(f) (BBHE, RMSHE), the drawback of these methods is obviously seen; that is, they preserves the mean brightness of the images without emphasizing the image details significantly; that is, the problem of intensity 


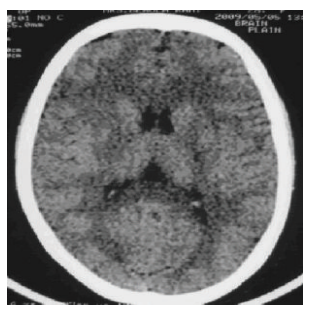

(a) Original

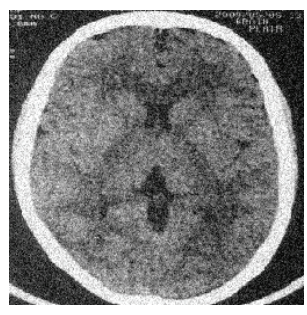

(b) $\mathrm{HE}$

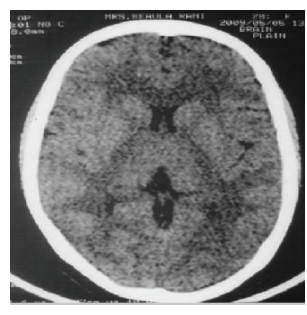

(c) BBHE

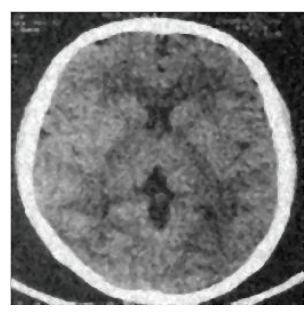

(d) DSIHE

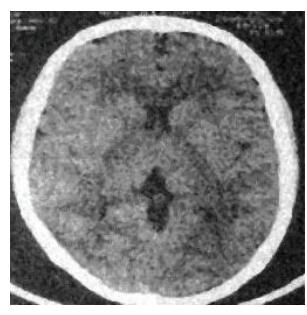

(e) MMBEBHE

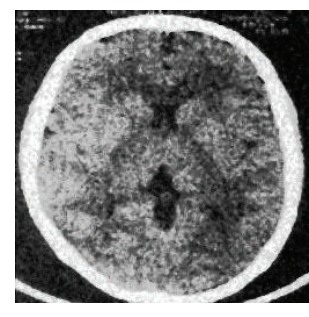

(f) RMSHE

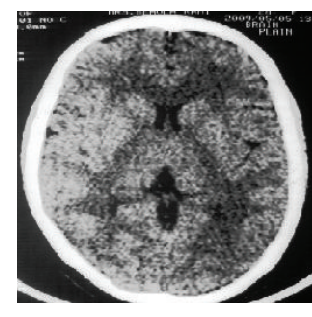

(g) BPDHE

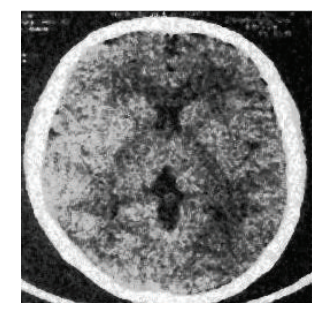

(h) BHEPL

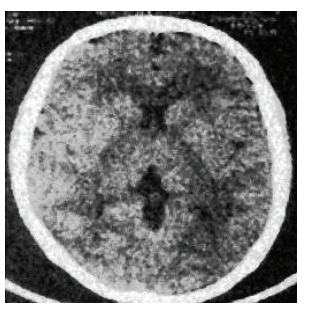

(i) Proposed QDAPLHE

FIGURE 4: Results for CT brain image.

TABLE 1: Absolute mean brightness error (AMBE).

\begin{tabular}{lcccccccc}
\hline Images & HE & BBHE & DSIHE & MMBEBHE & RMSHE $(r=2)$ & BPDHE & BHEPL & QDAPLHE \\
\hline Einstein & 17.17 & 19.27 & 12.07 & 14.27 & 10.17 & 3.65 & 3.07 & 0.45 \\
Girl & 5.29 & 23.51 & 4.46 & 3.04 & 25.4 & 0.13 & 0.07 \\
House & 58.81 & 25.09 & 31.92 & 25.06 & 8.07 & 5.43 & 3.57 & 2.54 \\
Couple & 96.42 & 33.17 & 43.81 & 18.45 & 10.28 & 3.56 & 2.78 \\
Copter & 62.72 & 17.21 & 26.91 & 32.14 & 3.08 & 2.54 & 1.24 & 0.22 \\
F16 & 49.72 & 1.09 & 13.5 & 15.31 & 1.24 & 1.54 & 2.53 \\
Jet & 71.76 & 4.91 & 26.84 & 2.07 & 0.64 & 0.29 & 0.37 & 1.89 \\
CT chest & 53.19 & 24.05 & 19.23 & 16.71 & 4.19 & 3.29 & 0.98 \\
CT brain & 62.08 & 34.21 & 29.71 & 19.31 & 5.84 & 3.56 & 1.97 & 0.34 \\
CT abdomen & 59.34 & 24.73 & 31.35 & 13.08 & 3.71 & 2.19 & 1.25 & 1.58 \\
Average & $\mathbf{5 3 . 6 5}$ & $\mathbf{2 0 . 7 2}$ & $\mathbf{2 3 . 9 8}$ & $\mathbf{1 5 . 9 4}$ & $\mathbf{4 . 7 8}$ & $\mathbf{2 . 8 5}$ & $\mathbf{1 . 7 9}$ & $\mathbf{1 . 1 9}$ \\
\hline
\end{tabular}

saturation occurs in some regions of the image even this methods improve the contrast of the image.

Figures 2(i), 3(i), and 4(i) show that the contrast of all the tested images is enhanced successfully using the proposed QDAPLHE method. In addition, this method preserves the image details successfully. Figures $2(\mathrm{~g}), 2(\mathrm{~h}), 3(\mathrm{~g}), 3(\mathrm{~h}), 4(\mathrm{~g})$, and $4(\mathrm{~h})$ show that BPDHE and BHEPL methods produce acceptable and natural enhanced images. Compared to these BPDHE and BHEPL methods, the proposed QDAPLHE method reduces the intensity saturation problem and also it can significantly improve the performance.

3.2. Quantitative Analysis. To prove the robustness of the proposed methods, three kinds of quantitative comparisons tests, the absolute mean brightness error, standard deviation, and peak signal to noise ratio, have been evaluated and tabulated in Tables $1-3$, respectively.

3.2.1. Absolute Mean Brightness Error (AMBE). The first test which is used as the performance measure is absolute mean brightness error (AMBE). AMBE is used to evaluate the ability of the enhancement method to maintain the mean brightness. One has

$$
\operatorname{AMBE}=\left(X_{m}-Y_{m}\right),
$$

where $X_{m}$ is the mean of the input image and $Y_{m}$ is the mean of the output image.

The minimum value of AMBE results in the fact that the mean brightness of the input is successfully maintained in the output image. Table 1 shows the AMBE measure obtained from the sample images.

The AMBE values calculated by the existing methods HE, $\mathrm{BBHE}$, and RMSHE are compared with the AMBE value of proposed method. From Table 1, it can be readily observed that the proposed method QDAPLHE has 13.13\% less AMBE average value when compared to BHEPL, the method with second minimum average value.

3.2.2. Standard Deviation (Image Contrast). By measuring the standard deviation, the contrast of the image can be studied. 
TABLE 2: Standard deviation (STD).

\begin{tabular}{lcccccccc}
\hline Images & HE & BBHE & DSIHE & MMBEBHE & RMSHE $(r=2)$ & BPDHE & BHEPL & QDAPLHE \\
\hline Einstein & 73.59 & 73.81 & 73.94 & 62.31 & 57.95 & 52.43 & 41.72 & 35.51 \\
Girl & 75.41 & 70.12 & 75.49 & 68.73 & 37.85 & 35.57 & 27.21 & 28.13 \\
House & 73.65 & 75.13 & 75.51 & 55.43 & 56.79 & 50.72 & 49.27 & 38.54 \\
Couple & 71.86 & 74.15 & 79.61 & 48.41 & 53.29 & 41.49 & 35.38 & 32.17 \\
Copter & 73.17 & 72.73 & 76.72 & 52.59 & 52.07 & 48.23 & 46.51 & 45.39 \\
F16 & 74.56 & 67.61 & 77.41 & 68.71 & 61.05 & 56.71 & 43.27 & 60.25 \\
Jet & 74.32 & 64.72 & 78.31 & 54.31 & 56.74 & 45.19 & 31.27 & 29.21 \\
CT chest & 85.12 & 74.19 & 81.29 & 56.23 & 49.16 & 42.75 & 35.19 & 34.31 \\
CT brain & 80.24 & 70.27 & 76.34 & 62.16 & 57.34 & 50.74 & 44.27 & 38.10 \\
CT abdomen & 82.34 & 76.35 & 79.56 & 69.13 & 50.37 & 48.29 & 45.84 & 29.74 \\
Average & $\mathbf{7 6 . 4 3}$ & $\mathbf{7 1 . 9 1}$ & $\mathbf{7 7 . 4 2}$ & $\mathbf{5 9 . 8 0}$ & $\mathbf{5 3 . 2 6}$ & $\mathbf{4 7 . 2 1}$ & $\mathbf{3 9 . 9 9}$ & $\mathbf{3 7 . 1 3}$ \\
\hline
\end{tabular}

TABLE 3: Peak signal to noise ratio (PSNR).

\begin{tabular}{lcccccccc}
\hline Images & HE & BBHE & DSIHE & MMBEBHE & RMSHE $(r=2)$ & BPDHE & BHEPL & QDAPLHE \\
\hline Einstein & 15.27 & 15.19 & 15.53 & 18.97 & 19.52 & 27.52 & 30.57 & 32.59 \\
Girl & 13.05 & 13.3 & 13.04 & 14.25 & 27.98 & 33.74 & 34.92 & 35.37 \\
House & 10.81 & 14.26 & 13.92 & 21.45 & 21.32 & 24.63 & 28.64 \\
Couple & 7.56 & 13.16 & 11.64 & 19.56 & 19.64 & 29.34 & 38.24 & 31.49 \\
Copter & 10.62 & 15.96 & 14.25 & 25.43 & 25.67 & 27.65 & 32.98 & 33.34 \\
F16 & 11.94 & 20.67 & 16.05 & 20.37 & 22.78 & 32.19 & 39.79 \\
Jet & 9.52 & 22.53 & 14.38 & 30.72 & 27.82 & 24.25 & 28.73 \\
CT chest & 14.35 & 19.53 & 15.37 & 29.73 & 33.46 & 31.26 & 34.29 & 31.22 \\
CT brain & 17.35 & 24.61 & 19.73 & 25.37 & 34.61 & 37.86 & 39.37 & 36.24 \\
CT abdomen & 16.54 & 23.25 & 18.16 & 23.45 & 31.23 & 32.74 & 33.78 & 31.27 \\
Average & $\mathbf{1 2 . 7 4}$ & $\mathbf{1 8 . 2 5}$ & $\mathbf{1 5 . 2 0}$ & $\mathbf{2 0 . 3 9}$ & $\mathbf{2 6 . 4 0}$ & $\mathbf{3 0 . 1 2}$ & $\mathbf{3 4 . 1 3}$ & $\mathbf{3 6 . 4 7}$ \\
\hline
\end{tabular}

Standard deviation " $\sigma$ " is given by

$$
\sigma=\sqrt{\sum_{l=0}^{L-1}(l-\mu) \times p(l),}
$$

where mean is given by

$$
\mu=\sum_{l=0}^{L-1} l \times p(l)
$$

and " $l$ " represents the pixel value in the image.

From Table 2, it is clear that the standard deviation value obtained by the proposed QDAPLHE method is less compared to all the existing methods for all the images.

3.2.3. Peak Signal to Noise Ratio (PSNR). Another quantitative test used to measure the richness of details and appropriateness is peak signal to noise ratio (PSNR). Based on mean squared errors (MSE), PSNR is defined as

$$
\text { PSNR }=\frac{10 \log _{10}(L-1)^{2}}{\text { MSE }}
$$

where

$$
\operatorname{MSE}=\frac{\sum_{i} \sum_{j}|X(i, j)-Y(i, j)|^{2}}{N}
$$

and $X(i, j)$ and $Y(i, j)$ are the input and output images, respectively. $N$ is the total number of pixels in the input or output images and $L$ is the number of intensity values.

The PSNR values for different images are tabulated in Table 3. From this table the PSNR values of three methods BPDHE, BHEPL, and QDAPLHE are ranked the first, second, and third highest values, respectively. It can be observed that the images processed by proposed QDAPLHE method produces the best PSNR values, as they are within the range $[31 \mathrm{~dB}-42 \mathrm{~dB}]$. From these values, it can be concluded that the proposed method performs image contrast enhancement and also produces images with a natural looking with less noise amplifying.

In overall, both the qualitative and the quantitative tests favor the proposed method QDAPLHE as the best among all the existing methods. Thus, it can be stated that the proposed algorithm produces the best image enhancement.

\section{Conclusion}

Although the histogram equalization is simple and effective algorithm for enhancement, it leads to overenhancement and intensity saturation problem. To overcome this effect, dynamic histogram equalization is powerful method for enhancing the low contrast images, and also in some cases 
it leads to noise amplification and intensity saturation problems. To overcome the level saturation effects occurring in histogram equalization, clipped histogram equalization methods are developed by restricting the enhancement rate. Hence, a new method QDAPLHE is proposed as a hybrid of dynamic histogram equalization method and clipped histogram equalization method. The qualitative and the quantitative analyses are performed on the proposed method and the results are represented in Figures 2-4 and Tables 1-3. From the experimental results, both the qualitative and the quantitative tests favor the proposed method QDAPLHE as the best among all the existing methods.

\section{Conflict of Interests}

The author declares that there is no conflict of interests regarding the publication of this paper.

\section{References}

[1] Y.-T. Kim, "Contrast enhancement using brightness preserving Bi-histogram equalization," IEEE Transactions on Consumer Electronics, vol. 43, no. 1, pp. 1-8, 1997.

[2] Y. Wang, Q. Chen, and B. Zhang, "Image enhancement based on equal area dualistic sub-image histogram equalization method," IEEE Transactions on Consumer Electronics, vol. 45, no. 1, pp. 68-75, 1999.

[3] S.-D. Chen and A. R. Ramli, "Minimum mean brightness error bi-histogram equalization in contrast enhancement," IEEE Transactions on Consumer Electronics, vol. 49, no. 4, pp. 13101319, 2003.

[4] S.-D. Chen and A. R. Ramli, "Contrast enhancement using recursive mean-separate histogram equalization for scalable brightness preservation," IEEE Transactions on Consumer Electronics, vol. 49, no. 4, pp. 1301-1309, 2003.

[5] K. S. Sim, C. P. Tso, and Y. Y. Tan, "Recursive sub-image histogram equalization applied to gray scale images," Pattern Recognition Letters, vol. 28, no. 10, pp. 1209-1221, 2007.

[6] M. A.-A. Wadud, M. H. Kabir, M. A. A. Dewan, and O. Chae, "A dynamic histogram equalization for image contrast enhancement," IEEE Transactions on Consumer Electronics, vol. 53, no. 2, pp. 593-600, 2007.

[7] H. Ibrahim and N. S. P. Kong, "Brightness preserving dynamic histogram equalization for image contrast enhancement," IEEE Transactions on Consumer Electronics, vol. 53, no. 4, pp. 17521758, 2007.

[8] N. R. S. Parveen and M. M. Sathik, "Enhancement of bone fracture images by equalization methods," in Proceedings of the International Conference on Computer Technology and Development, pp. 391-394, November 2009.

[9] K. Y. Pang, L. lznita, A. Fadzil, and A. N. Hanung, "Segmentation of retinal vasculature in colour fundus images," in Proceedings of the Innovative Technologies in Intelligent Systems and Industrial Applications (CITISIA '09), pp. 398-401, July 2009.

[10] K. Kokufuta and T. Maruyama, "Real-time processing of contrast limited adaptive histogram equalization on FPGA," in Proceedings of the 20th International Conference on Field Programmable Logic and Applications (FPL '10), pp. 155-158, September 2010.
[11] X. Wu, "A linear programming approach for optimal contrasttone mapping," IEEE Transactions on Image Processing, vol. 20, no. 5, pp. 1262-1272, 2011.

[12] C. H. Ooi, N. S. P. Kong, and H. Ibrahim, "Bi-histogram equalization with a plateau limit for digital image enhancement," IEEE Transactions on Consumer Electronics, vol. 55, no. 4, pp. 2072-2080, 2009.

[13] P. Wayalun, "Images enhancement of G-band chromosome using histogram equalization, OTSU thresholding, morphological dilation and flood fill techniques," in Proceedings of the 8th IEEE International Conference on Computing and Networking Technology (ICCNT '12), pp. 163-168, 2012.

[14] N. bt Shamsuddin, W. F. bt Wan Ahmad, B. B. Baharudin, M. K. B. M. Rajuddin, and F. bt Mohd, "Significance level of image enhancement techniques for underwater images," in Proceedings of the IEEE International Conference on Computer \& Information Science (ICCIS '12), vol. 1, pp. 490-494, June 2012.

[15] A. K. Bhandari, A. Kumar, and G. K. Singh, "SVD based poor contrast improvement of blurred multispectral remote sensing satellite images," in Proceedings of the 3rd IEEE International Conference on Computer and Communication Technology (ICCCT '12), pp. 156-159, Allahabad, India, November 2012.

[16] K. Saranya, B. Ramasubramanian, and S. Kaja Mohideen, "A novel approach for the detection of new vessels in the retinal images for screening diabetic retinopathy," in Proceedings of the International Conference on Communication and Signal Processing (ICCSP '12), pp. 57-61, April 2012.

[17] C.-Y. Tsai, "A fast dynamic range compression with local contrast preservation algorithm and its application to real-time video enhancement," IEEE Transactions on Multimedia, vol. 14, no. 4, pp. 1140-1152, 2012.

[18] M. Karaman, L. Onural, and A. Atalar, "Design and implementation of a general-purpose median filter unit in CMOS VLSI," IEEE Journal of Solid-State Circuits, vol. 25, no. 2, pp. 505-513, 1990.

[19] N. Sengee and H. K. Choi, "Brightness preserving weight clustering histogram equalization," IEEE Transactions on Consumer Electronics, vol. 54, no. 3, pp. 1329-1337, 2008.

[20] S. M. Pizer, R. E. Johnston, J. P. Ericksen, B. C. Yankaskas, and K. E. Muller, "Contrast-limited adaptive histogram equalization: speed and effectiveness," in Proceedings of the 1st Conference on Visualization in Biomedical Computing (VBC '90), pp. 337-345, May 1990. 


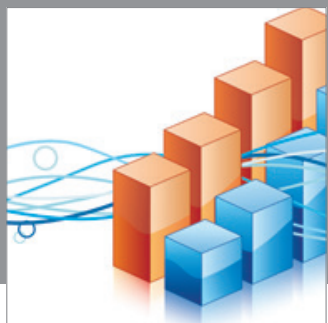

Advances in

Operations Research

mansans

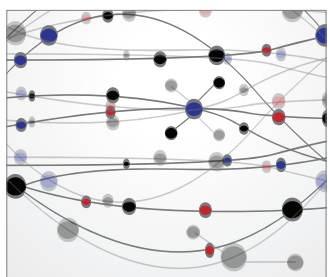

The Scientific World Journal
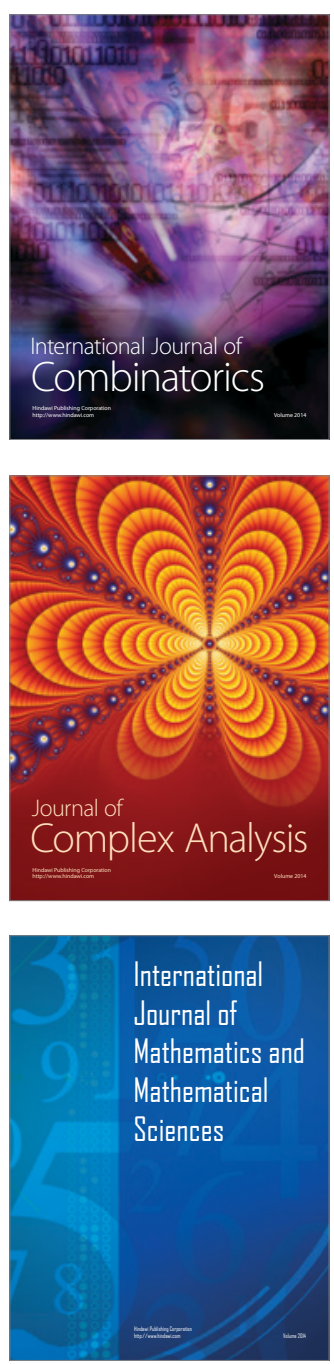
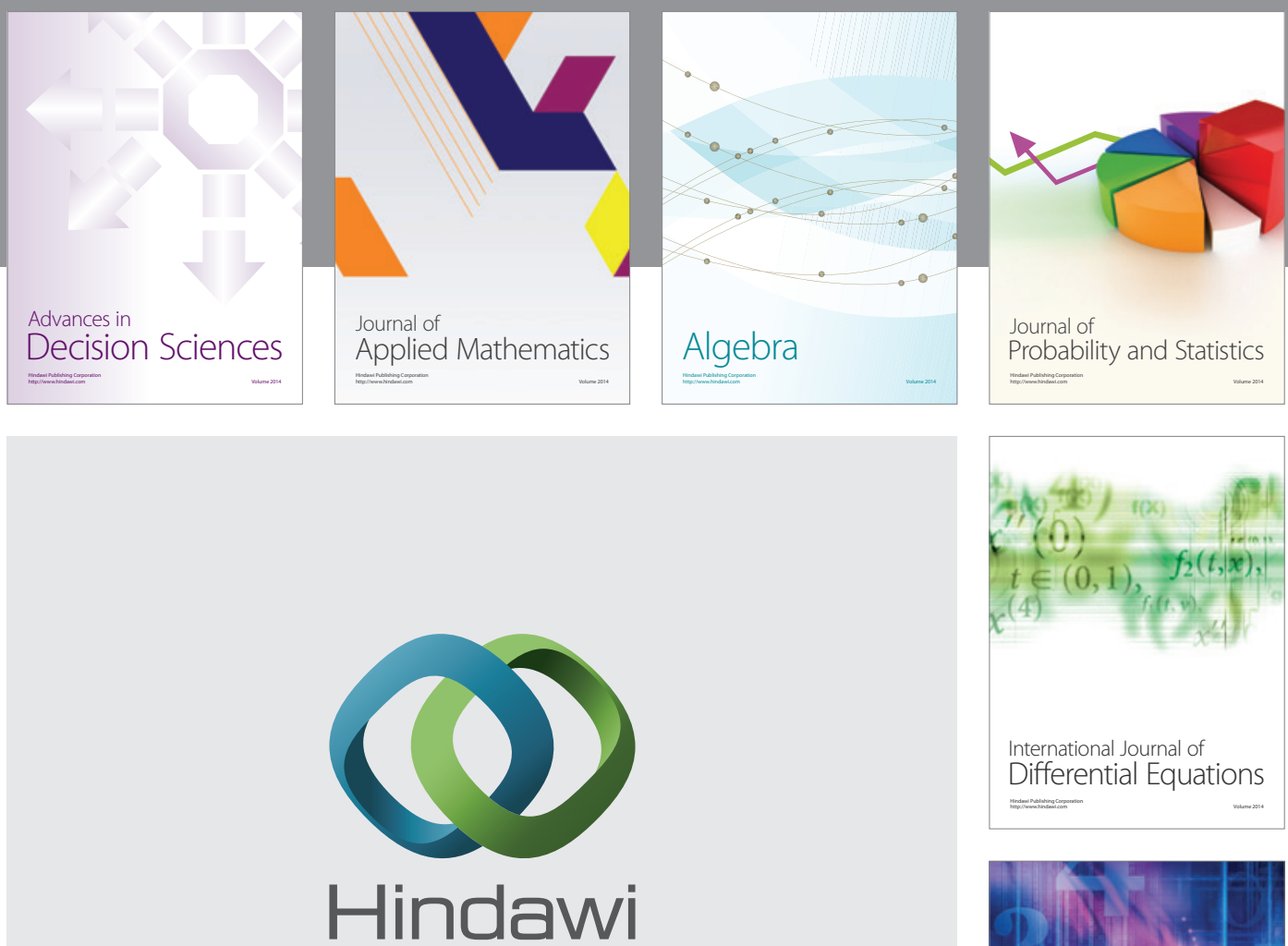

Submit your manuscripts at http://www.hindawi.com
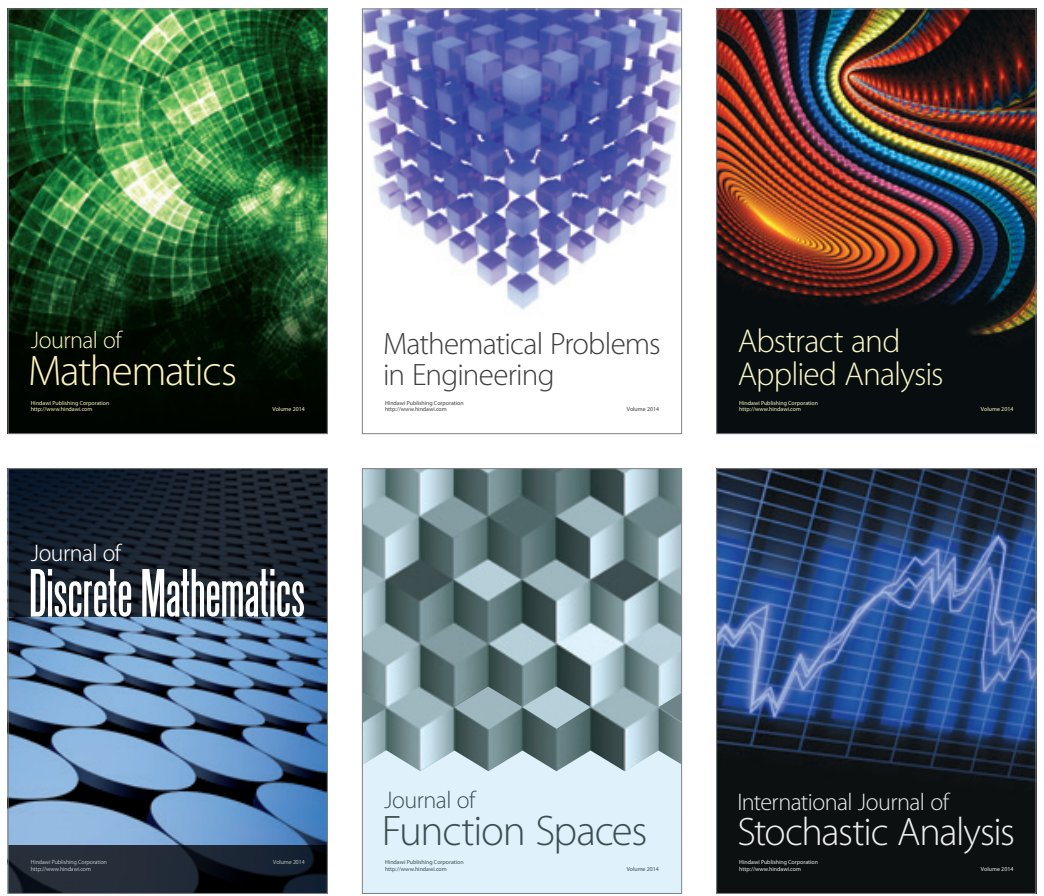

Journal of

Function Spaces

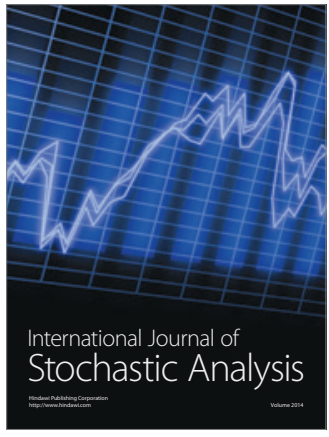

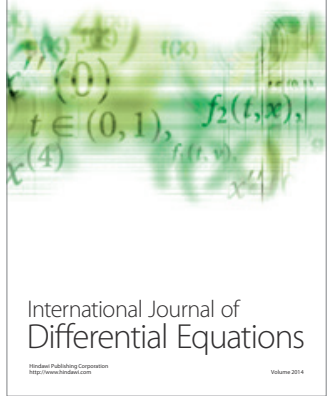
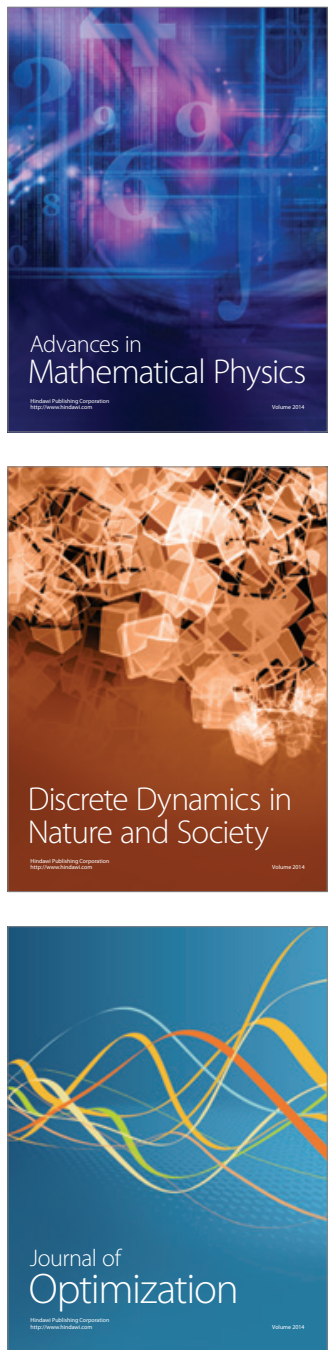